\title{
NRP1-positive lung cancer cells possess tumor-initiating properties
}

\author{
LUIS E. JIMENEZ-HERNANDEZ ${ }^{1}$, KARLA VAZQUEZ-SANTILLAN ${ }^{1}$, ROSARIO CASTRO-OROPEZA ${ }^{1}$, \\ GUSTAVO MARTINEZ-RUIZ ${ }^{3,4}$, LAURA MUÑOZ-GALINDO ${ }^{1}$, CAROLINA GONZALEZ-TORRES ${ }^{1}$, \\ CARLO C. CORTES-GONZALEZ ${ }^{2}$, GEORGINA VICTORIA-ACOSTA ${ }^{1}$, \\ JORGE MELENDEZ-ZAJGLA $^{1}$ and VILMA MALDONADO ${ }^{1}$ \\ ${ }^{1}$ National Institute of Genomic Medicine; ${ }^{2}$ National Institute of Cancerology; ${ }^{3}$ Federico Gomez Children's Hospital; \\ ${ }^{4}$ School of Medicine, National Autonomous University of Mexico, Mexico City, Mexico
}

Received June 2, 2017; Accepted October 13, 2017

DOI: $10.3892 / o r .2017 .6089$

\begin{abstract}
Tumor-initiating cells possess the capacity for self-renewal and to create heterogeneous cell lineages within a tumor. Therefore, the identification and isolation of cancer stem cells is an essential step in the analysis of their biology. The aim of the present study was to determine whether the cell surface protein neuropilin 1 (NRP1) can be used as a biomarker of stem-like cells in lung cancer tumors. For this purpose, NRP1-negative (NRP1 ${ }^{-}$) and NRP1-positive (NRP1 $\left.1^{+}\right)$ cell subpopulations from two lung cancer cell lines were sorted by flow cytometry. The $\mathrm{NRP1}^{+}$cell subpopulation showed an increased expression of pluripotency markers OCT-4, Bmi-1 and NANOG, as well as higher cell migration, clonogenic and self-renewal capacities. NRP1 gene knockdown resulted not only in a decreased expression of stemness markers but also in a decrease in the clonogenic, cell migration and self-renewal potential. In addition, the $\mathrm{NRP}^{+}$cell subpopulation exhibited dysregulated expression of epithelial-to-mesenchymal transition-associated genes, including the $\Delta \mathrm{Np} 63$ isoform protein, a previously reported characteristic of cancer stem cells. Notably, a genome-wide expression analysis of NRP1-knockdown cells revealed a potential new NRP1 pathway involving OLFML3 and genes associated with mitochondrial function. In conclusion, we demonstrated that $\mathrm{NRP}^{+}$lung cancer cells have tumor-initiating properties. NRP1 could be a useful biomarker for tumor-initiating cells in lung cancer tumors.
\end{abstract}

\section{Introduction}

Lung cancer, with an estimated patient survival rate of $\sim 5$ years, has become a leading cause of cancer-related death (1). It has been proposed that tumors are sustained by a cell subpopulation with tumor-initiating properties. These cells, known as

Correspondence to: Dr Vilma Maldonado, National Institute of Genomic Medicine, Mexico City 14610, Mexico

E-mail:vmaldonado@inmegen.gob.mx

Key words: tumor-initiating cells, lung cancer, neuropilin 1, olfactomedin-like 3, tumor protein p63, miRNA tumor-initiating cells (TICs) or cancer stem cells (CSCs), have self-renewal capacity and the ability to create the characteristic cellular heterogeneity found in solid tumors. TICs have been previously identified and isolated using specific cell membrane markers, such as OV-6, CD13 and CD44 in liver cancer (2) and EpCAM, CD44, CD24 in breast cancer (3).

In 2008, Eramo et al identified for the first time tumor-initiating cells from lung tumors using CD133 as a biomarker (4-6). However, this result has been disputed by several authors. For example, in 2009, Meng et al found that both CD133 and $\mathrm{CD}_{133^{+}}$cell populations from lung cancer possess TIC properties (7), and more recently Qiu et al found no statistical difference between the ability of $\mathrm{CD} 133^{-}$and $\mathrm{CD} 133^{+}$cell populations to form pneumospheres $(8,9)$. The predictive value to detect this subpopulation in lung cancer cell lines of other TIC biomarkers, including ALDH1 and CD24, remains controversial (10-12). To date, there are no reliable biomarkers for the detection of tumor-initiating cells in lung cancer.

Neuropilin 1 (NRP1) is a transmembrane glycoprotein involved in various cellular processes that include angiogenesis, cell migration, $\mathrm{T}$ cell activation, survival and axon growth $(13,14)$. Existing data suggest an association between NRP1 expression and a tumor-initiating cell phenotype. For example, endothelial progenitors can be identified by NRP1 expression (15). In addition, it has been shown that NRP1 is essential for proliferation and cell migration of adult mesenchymal stem cells (16). NRP1 promotes TIC-related cellular processes, such as angiogenesis, cell migration, invasion and metastasis in cancer tissue $(17,18)$. Moreover, NRP1 overexpression induces a poorly differentiated phenotype in renal carcinoma cells (19). Furthermore, NRP1 also maintains a tumor-initiating phenotype in glioma and skin cancer cells (20). In addition, Barr et al reported that NRP1 is a critical co-receptor in VEGF-mediated survival and tumor growth of NSCLC cells (21).

In the present study, we analyzed whether NRP1 expression was able to identified a TIC subpopulation in lung cancer cell lines and is involved in the maintenance of these cells. We found that NRP1-expressing cells exhibited TIC-like properties, i.e. stemness and high clonogenic capability. Concordant with this, NRP1 downregulation inhibited the expression of stemness markers and prevented cell migration and pneumosphere formation. Finally, a genome-wide expression analysis in 
NRP1-knockdown cells revealed differentially expressed genes that could be involved in the maintenance of the TIC phenotype.

\section{Materials and methods}

Cell culture. Lung cancer cell lines A549 and Calu-1 were obtained from the American Type Culture Collection (CCL-185 and HTB-54; ATCC; Manassas, VA, USA). Cell lines were maintained in Dulbecco's modified Eagle's medium (DMEM) (Corning Life Sciences, Corning, NY, USA) supplemented with 5\% fetal bovine serum (FBS) (\#30-2020; ATCC), and kept at $37^{\circ} \mathrm{C}$, with $5 \% \mathrm{CO}_{2}$ and $95 \%$ humidity.

Flow cytometry. Cells were detached from the plates using StemPro Accutase (Thermo Scientific, Waltham, MA, USA), washed with $1 \mathrm{X}$ phosphate-buffered saline (PBS), and suspended in $1 \%$ FBS. Subsequently, $1 \times 10^{7}$ cells were incubated in ice with the antibodies APC-NRP1 (130-090-900) at a 1:10 dilution for $40 \mathrm{~min}$. The isotype control antibodies IgG1-APC (130-092-214) were used. All antibodies were obtained from Miltenyi Biotech (Bergisch Gladbach, Germany). The cells were sorted with a FACSAria flow cytometer (Becton-Dickinson, Franklin Lakes, NJ, USA), according to their phenotype into NRP1-negative (NRP1 ${ }^{-}$) and NRP1-positive $\left(\mathrm{NRP}^{+}\right)$subpopulations. All of the sorted populations were maintained under standard growth conditions.

Semi-quantitative PCR analysis. Total RNA was extracted from the cells using TRIzol reagent (Thermo Fisher Scientific, Waltham, MA USA) following the manufacturer's instructions. RNA concentration and purity were determined using a NanoDrop (Thermo Scientific, Wilmington, DE, USA). Subsequently, $1 \mu \mathrm{g}$ of total RNA was reverse transcribed to cDNA using the High Capacity cDNA reverse transcription kit (Applied Biosystems, Foster City, CA, USA) in a total volume of $20 \mu \mathrm{l}$. The cDNA was amplified by semi-quantitative PCR using specific primers for each tested gene. Saturation curves for each amplified fragment was carried out at different cycles. TATA-box binding protein (TBP) or $18 \mathrm{~S}$ gene expression were used as an internal reference control. PCR products were resolved by electrophoresis on a $1.5 \%$ agarose gel. The DNA bands were visualized using Gel-Red staining (Biotium, Hayward, CA, USA).

Colony forming assays. To evaluate the clonogenic capacity of each isolated population, $4 \times 10^{3}$ cells were plated in DMEM with $0.3 \%$ low melting point agarose and 10\% FBS (as the upper layer). As a basecoat, a mixture of $1 \%$ low-melting agarose with $2 \mathrm{X}$ DMEM medium in a 1:1 ratio was used. The cells were incubated for 4 weeks at $37^{\circ} \mathrm{C}$ with $5 \% \mathrm{CO}_{2}$ and 95\% humidity. To prevent the agar plate from dehydrating, $200 \mu$ l of DMEM with $10 \%$ FBS was added every 3 days. At the end of the incubation period, the colonies were stained with $0.01 \%$ crystal violet and counted using an inverted microscope (Olympus, Inc., New Orleans, LA, USA).

Pneumosphere formation assay. To assess the ability of each population to form pneumospheres, $5 \times 10^{4}$ cells were seeded in $12.5 \mathrm{~cm}^{2}$ flasks in Leibovitz's medium (both from Corning Inc., Corning, NY, USA), supplemented with 5\% FBS. Cells were maintained at $37^{\circ} \mathrm{C}$ with a constant $50 \mathrm{rpm}$ orbital agitation for several days. Medium was changed every day. After 5 days, the pneumospheres were counted and dissociated into a single-cell suspension using $2.5 \%$ trypsin (Thermo Fisher Scientific). The cells were counted and seeded into new flasks for the following serial passage assays.

NRP1 knockdown. Two shRNAs targeting NRP1 (shNRP1) were designed using the online tool from Clontech Laboratories, Inc. (Mountain View, CA, USA) (http://www.clontech.com). The shRNA oligonucleotides were annealed and ligated into the vector pSIREN-RetroQ (631526; Clontech Laboratories, Inc.) using the Quick Ligation kit from Fermentas (K142; Waltham, MA, USA). An shRNA targeting the luciferase gene (shLuc) was used as control. Lipofectamine 2000 (Thermo Fisher Scientific) was used in the transfection assays as instructed by the manufacturer. Positive selection of A549 cells was performed with $7.5 \mu \mathrm{g} / \mathrm{ml}$ puromycin (Sigma-Aldrich, St. Louis, MO, USA), whereas Calu-1 cells were selected with $5 \mu \mathrm{g} / \mathrm{ml}$ puromycin for 2 weeks. The gene expression level of NRP1 in the transfected cells was analyzed by semi-quantitative PCR.

Western blot analysis. A549 cells were washed with 1X PBS, harvested and centrifuged at $1,000 \mathrm{rpm}$ for $5 \mathrm{~min}$; the cell pellets were resuspended in $300 \mu \mathrm{l}$ of buffer A (1X protease inhibitor, $10 \mathrm{mM}$ HEPES pH 7.5, $15 \mathrm{mM} \mathrm{KCl}, 0.1 \mathrm{mM}$ EDTA, $0.1 \mathrm{mM}$ EGTA and $2 \mathrm{mM} \mathrm{MgCl}_{2}$ ), incubated on ice for $15 \mathrm{~min}$, after which $0.01 \% \mathrm{NP} 40$ was added for $1 \mathrm{~min}$. The lysates were centrifuged at $6,000 \mathrm{rpm} / 5 \mathrm{~min}$. The cytosolic fraction (supernatant) was recovered and stored at $-20^{\circ} \mathrm{C}$ for later analysis. The remaining pellet was resuspended in $200 \mu 1$ of buffer C ( $400 \mathrm{mM} \mathrm{NaCl}, 1 \mathrm{mM}$ EDTA, $25 \mathrm{mM}$ HEPES pH 7.5 and $20 \%$ glycerol) and incubated for $30 \mathrm{~min}$ on ice. After incubation, the lysates were centrifuged at $8,000 \mathrm{rpm} / 5 \mathrm{~min}$ and the nuclear fraction (supernatant) was recovered and stored at $-20^{\circ} \mathrm{C}$. Fractions were resolved by sodium dodecyl sulfate-polyacrylamide gel electrophoresis (SDS-PAGE), transferred to polyvinylidene difluoride (PVDF) membranes (Millipore, Bedford, MA, USA) and blocked with 5\% albumin in Tris-buffered saline (TBS) with $0.1 \%$ Tween for $1 \mathrm{~h}$. The membranes were then incubated with the primary antibody overnight at $4{ }^{\circ} \mathrm{C}$. Antibodies were diluted in $5 \%$ bovine serum albumin, $1 \mathrm{X}$ TBS and $0.1 \%$ Tween. For the NRP1 antibody (36-1400; Invitrogen, Waltham, MA, USA) a concentration of $2 \mu \mathrm{g} / \mathrm{ml}$ was used. For p63 and phospho-p63 antibodies (\#4892 and \#4981; Cell Signaling Technology Inc., Danvers, MA USA) a dilution of 1:1,000 was used. As loading controls, lamin B (sc-374015) and tubulin (sc-53646) (Santa Cruz Biotechnology, Dallas, TX, USA) were used. The membranes were washed with TBS-Tween $0.2 \%$ and incubated with the secondary antibodies, anti-mouse IgG HRP-conjugated W402B or anti-rabbit IgG HRP-conjugated W401B (Promega, Madison, WI, USA) at room temperature for $1 \mathrm{~h}$. Proteins were detected using the Western Kit Immobilon (Millipore) and visualized with VersaDoc Imaging System (Bio-Rad, Hercules, CA, USA).

Wound healing assay. Cell migration capacity was determined by seeding $1 \times 10^{6}$ cells into 6 -well plates (Corning) with 
A

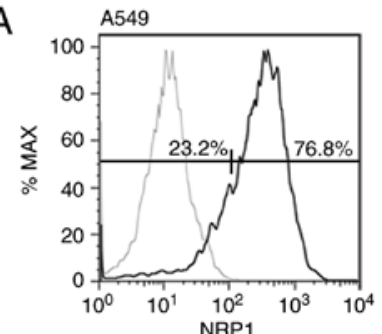

C

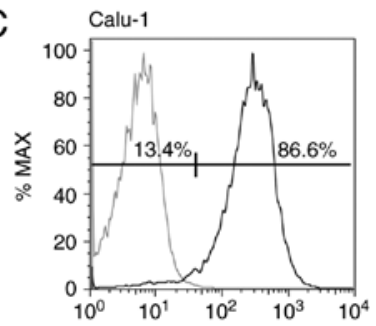

B

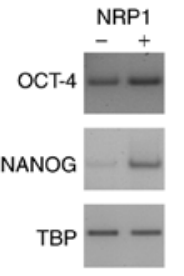

D

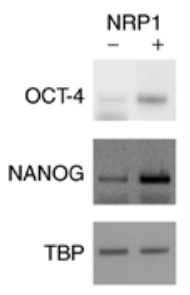

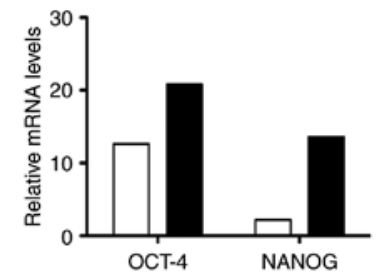

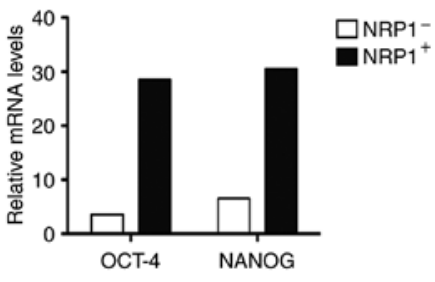

Figure 1. Identification of NRP1 ${ }^{+}$cells and analyses of expression of pluripotency markers. (A) Representative flow cytometry histograms of A549 cells labeled for NRP1 (APC); the percentage of each population (NRP1' and NRP1 ${ }^{+}$) is indicated. (B) Expression of stemness markers in NRP1- and NRP ${ }^{+}$A549 cells. (C) Representative flow cytometry histograms of Calu-1 cells labeled for NRP1 (APC, left panel); the percentage of each population (NRP1 ${ }^{-}$and NRP1 ${ }^{+}$) is indicated. (D) Expression of stemness markers in NRP1 ${ }^{-}$and $\mathrm{NRP}^{+} \mathrm{Calu}-1$ cells. TBP was used as a normalization control.

DMEM and 5\% FBS. After $12 \mathrm{~h}$, a wound was made to the confluent cell layer using a $200-\mu 1$ pipette tip. Images of the wounded area were captured at 0,24 and $48 \mathrm{~h}$. Cell migration capacity was calculated determining the wound opening area using ImageJ software (22).

Microarray analysis. Total RNA from shNRP1- and shLuc-transfected cells was extracted from 3 independent experiments. RNA integrity was assessed on a Bioanalyzer 2100 (Agilent Technologies, Inc., Santa Clara, CA, USA), and samples with a RNA integrity number (RIN) $>9$ were used. Microarray experiments were performed using the Affymetrix GeneChip platform (HuGene 2.0 ST) in the National Institute of Genomic Medicine's Microarray High Technology Core Center, Mexico. Raw data were normalized using Robust Multi-array Average (RMA) (23). Significance of the differential expression was calculated with one-way analysis of variance (ANOVA). Genes with $\mathrm{P}<0.05$ and a fold change higher or lower than 1.5 were considered for further analysis.

Immunofluorescence. A549 and Calu-1 cell lines were culture over glass coverslips in 6-well plates for $24 \mathrm{~h}$ after cell transfection. Cells were rinsed with PBS and fixed with $4 \%$ paraformaldehyde (PFA) (Sigma-Aldrich) for $30 \mathrm{~min}$. Subsequently, cells were blocked in PBS and $5 \%$ bovine serum albumin (Sigma-Aldrich) during $2 \mathrm{~h}$ and were then incubated overnight with the NRP1 primary antibody (Thermo-Fisher, Waltham, MA USA). Cells were washed and incubated with a Cy3 linked secondary antibody (AP124C; Millipore) for $1 \mathrm{~h}$. Finally, cells were washed with PBS and slides were mounting in EverBrite mounting medium with 4,6-diamidino-2-phenylindole (DAPI) (Biotium) and stored at $4^{\circ} \mathrm{C}$. Fluorescence analysis was performed on a confocal microscope (Zeiss LSM 510; Carl Zeiss, Jena, Germany).

Cell migration and invasion assays. The cell migration ability of A549 and Calu-1 NRP1-deficient cells was examined using Transwell polycarbonate permeable supports (BD Biosciences,
Franklin Lakes, NJ, USA). A total of $3.8 \times 10^{4}$ cells in $300 \mu \mathrm{l}$ of culture medium without FBS were placed in the upper chamber and $700 \mu \mathrm{l}$ of medium with $10 \%$ FBS were added to the lower chamber. Cells were culture for $24 \mathrm{~h}$, and then the inserts were removed from the wells. Cells on the upper surface of the membrane were removed and migrating cells located on the lower surface were washed with PBS and fixed with $4 \%$ PFA and finally stained with $0.1 \%$ crystal violet. Images were captured in a stereomicroscope and the number of migrating cells were counted with ImageJ software (24).

Cell proliferation assays. Cell proliferation of A549 and Calu-1 NRP1-deficient cells was analyzed using an MTS method. Briefly, $5 \times 10^{3}$ transiently transfected cells were seeded in triplicates onto 96 -well plates and incubated at $37^{\circ} \mathrm{C}$ under a $5 \% \mathrm{CO}_{2}$ atmosphere. Forty-eight hours after transfection, cells were cultured for 24,48 and $72 \mathrm{~h}$. On the indicated days, cell numbers were measured in triplicate using the CellTiter-96 Non-Radioactive Cell Proliferation Assay kit (Promega). A549 and Calu-1 cells were incubated with the MTS reagent during 1 and $2 \mathrm{~h}$, respectively. Then, absorbance was measured at an optical density of $590 \mathrm{~nm}$ using a multi-detector reader (Beckman Coulter, Inc., Brea, CA, USA). Proliferation curves were constructed by calculating the mean value of optical density measurements in each condition.

Statistical analysis. FACS data were analyzed using the FlowJo 8.7 software (TreeStar, Inc., Ashland, OR, USA). One-way ANOVA and a t-test with Bonferroni correction were applied. Analysis of the microarray data was performed using the Bioconductor software.

\section{Results}

Isolation and expression of pluripotency markers in $\mathrm{NRPI}^{+}$ lung cancer cells. The percentage of NRP1+ cells in A549 and Calu-1 lung cancer cell lines was determined using flow cytometry. As shown in Fig. 1A and C, NRP1 was present 

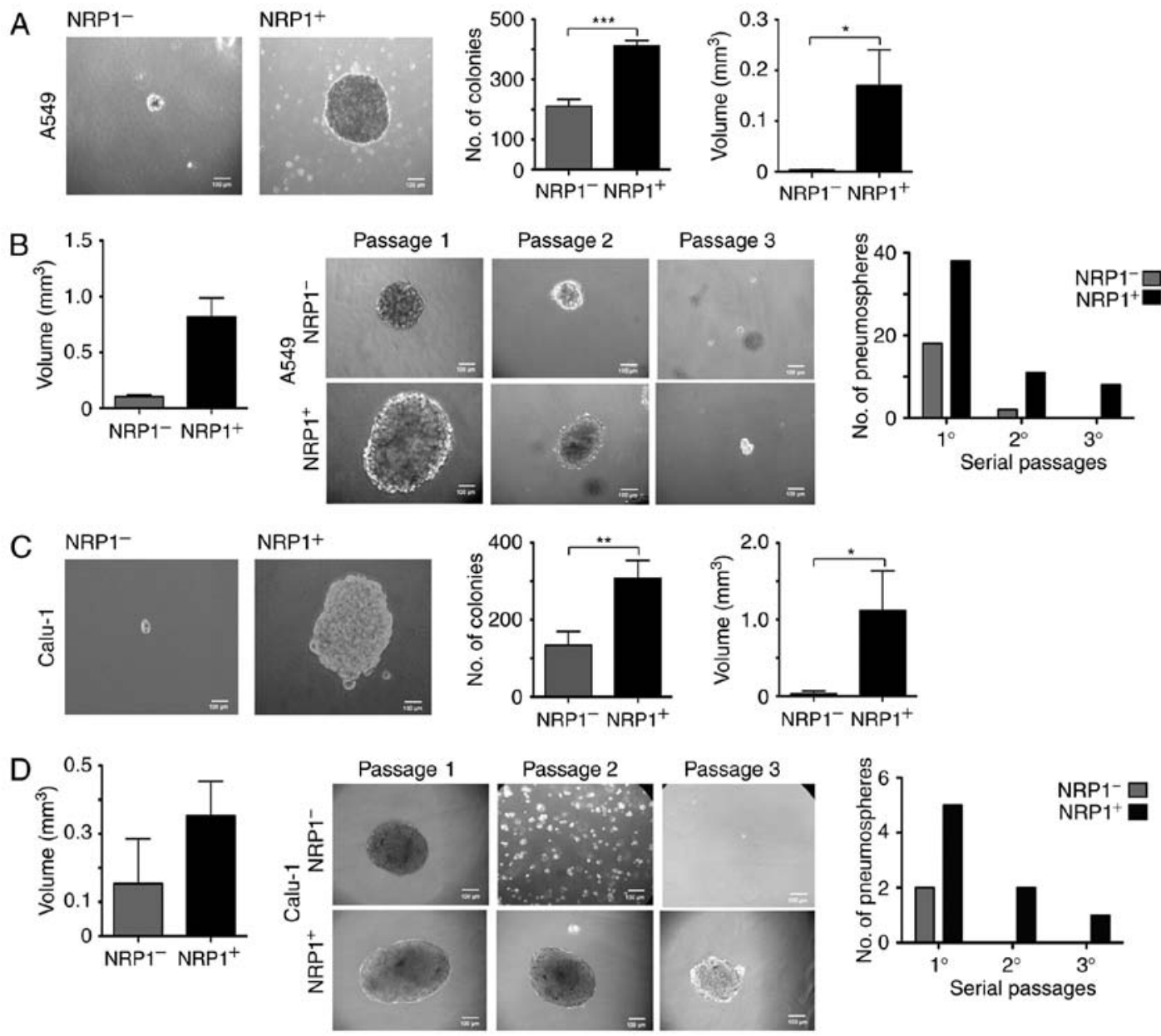

Figure 2. Clonogenic capacity and renewal of $\mathrm{NRP}^{+}$cells. (A) Colonies formed by NRP1 ${ }^{-}$and $\mathrm{NRP}^{+} \mathrm{A} 549$ cells. (B) Pneumosphere formation ability of $\mathrm{NRP1}^{-}$and NRP1 ${ }^{+}$A549 cells. (C) Colonies formed by NRP1 ${ }^{-}$and NRP1 ${ }^{+}$Calu-1 cells. (D) Pneumosphere-forming capacity of NRP1 and NRP1 ${ }^{+}$Calu-1 cells Data represent the average of 3 experiments $\left({ }^{*} \mathrm{P}<0.1,{ }^{* * *} \mathrm{P}<0.005,{ }^{* * *} \mathrm{P}<0.001\right)$.

in $76.8 \pm 13 \%$ of A549 and $86.6 \pm 4 \%$ of Calu- 1 cells. We then analyzed key stemness markers in the sorted $\mathrm{NRP}^{+}$and NRP1 $1^{-}$subpopulations. $\mathrm{NRP}^{+}$subpopulations of both cell lines presented higher OCT-4 and NANOG expression when compared with NRP1 ${ }^{-}$cells, as assessed by semi-quantitative PCR (Fig. 1B and D).

$\mathrm{NRPI}^{+}$cells exhibit high clonogenic capacity and selfrenewal ability. In order to assess whether $\mathrm{NRP}^{+}$cells exhibit TIC abilities, we compared the clonogenic and self-renewal capacities of enriched $\mathrm{NRP}^{+}{ }^{+}$and NRP1 ${ }^{-}$subpopulations using a combination of soft agar and serial-passage pneumosphere formation assays. As shown in Fig. 2A and $\mathrm{C}, \mathrm{NRP} 1^{+}$cells presented higher clonogenic capacity (represented by colony number and size) compared with NRP1 ${ }^{-}$cells in both A549 and Calu- 1 cell lines. Similarly, NRP1 $1^{+}$cells derived from both A549 and Calu-1 cell lines exhibited a higher selfrenewal capacity, as assessed by serial-passage pneumosphere assays (Fig. 2B and D).

$\mathrm{NRPI}^{+}$cells express additional stemness markers. To further support our hypothesis that $\mathrm{NRP}^{+}$cells represent an enriched lung TIC subpopulation, we analyzed an expression panel of stemness markers $(24,25)$. As shown in Fig. 3A, the NRP1 ${ }^{+}$ subpopulation had higher expression of Bmi-1, ALDH-1,
KRT-5, CD133 and p63 than the NRP1- subpopulation derived from both cell lines (Fig. 3A).

It has been reported that epithelial-mesenchymal transition (EMT) is associated with the generation and maintenance of TICs (26). Concordant with this, both A549 and Calu-1 $\mathrm{NRP}^{+}$cell subpopulations presented higher Snail and vimentin expression when compared to the NRP1 ${ }^{-}$subpopulations (Fig. 3B).

NRPI expression is associated with a TIC phenotype in lung cancer cells. Seeking to further establish the association between NRP1 expression and a stem cell phenotype, we performed loss-of-function experiments by means of NRP1 gene knockdown. The decrease in NRP1 expression was confirmed by RT-PCR (Fig. 3C). Downregulated NRP1 expression was accompanied by a concomitant decrease in OCT-4 and Bmi-1 expression, when compared to the shLuc control cells (Fig. 3C). Likewise, NRP1 knockdown also decreased the expression of p63 and its $\triangle$ Np63 mRNA isoform (Fig. 3D, left panel). Downregulation of p63 was corroborated at the protein level (Fig. 3D, right panel). We found that total p63 and phosphorylated p63 (P-p63) proteins were predominantly expressed in the nuclear fraction. Furthermore, nuclear P-p63 was detected at lower levels in the A459 cells with silenced NRP1 expression when compared to the control cells. 

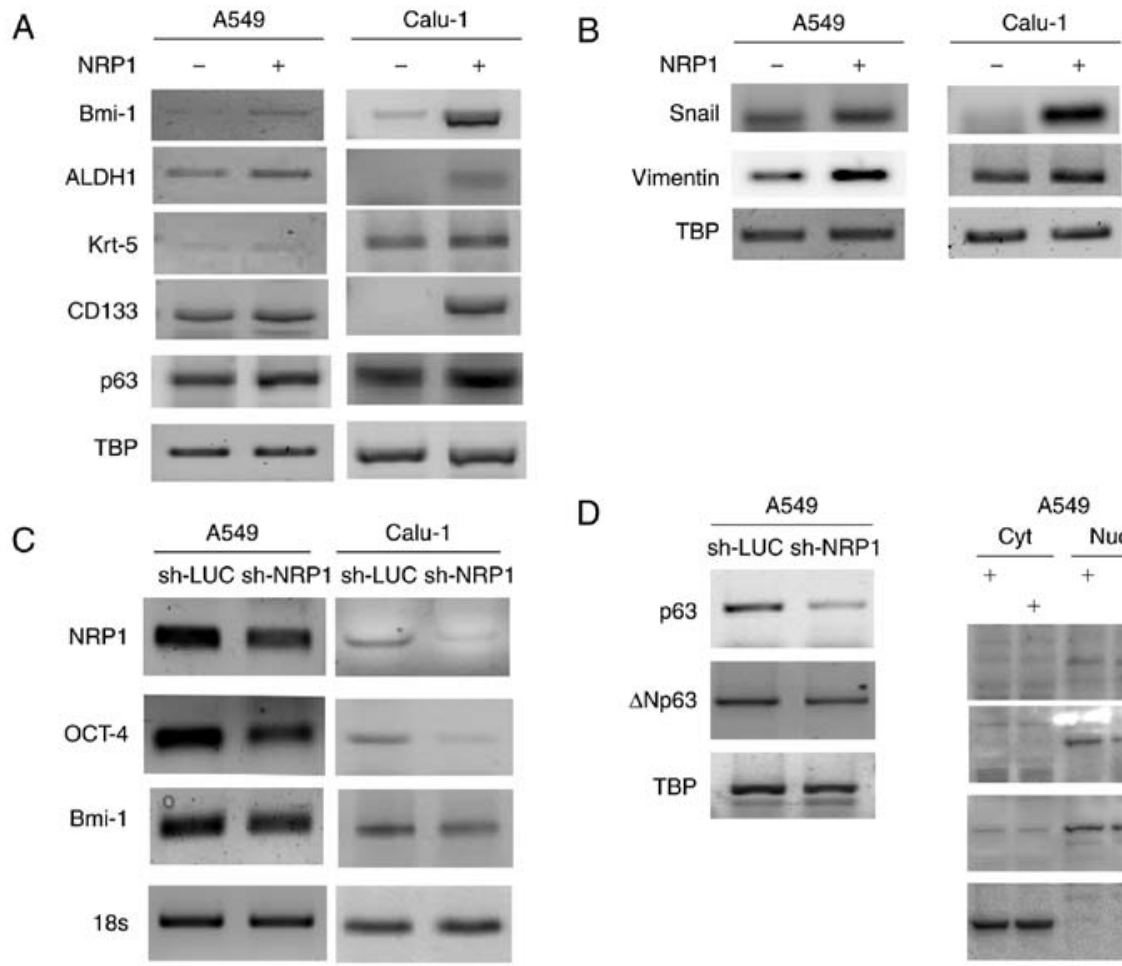

D
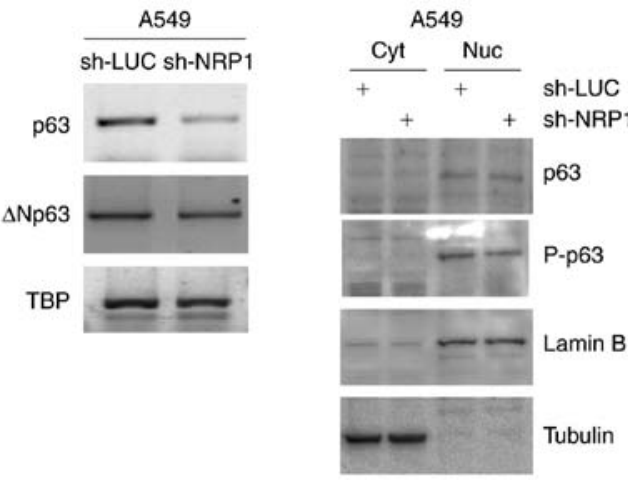

Figure 3. Expression of lung stemness and EMT markers in A549 and Calu-1 cell lines. (A) Stemness marker expression in NRP1 and NRP1 ${ }^{+}$subpopulations. (B) Expression of EMT markers in NRP1 ${ }^{-}$and NRP1 ${ }^{+}$cells. (C) Expression of stemness markers in A549 and Calu-1 cells transfected with sh-NRP1. (D) Expression of p63 and DNp63 isoform (left panel) and western blotting of p63 and P-p63 (right panel) in cytosolic and nuclear fractions of A549 cells transfected with sh-NRP1 or sh-LUC. All assays were performed by semi-quantitative RT-PCR. TBP or 18s was used as a normalization control.
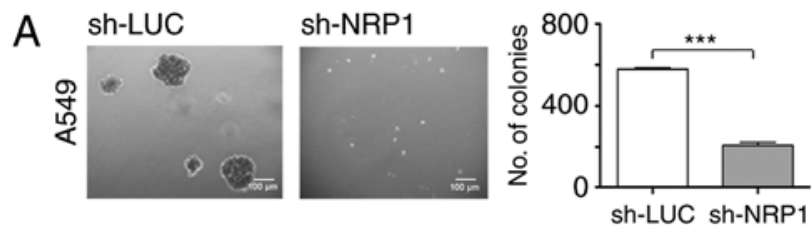
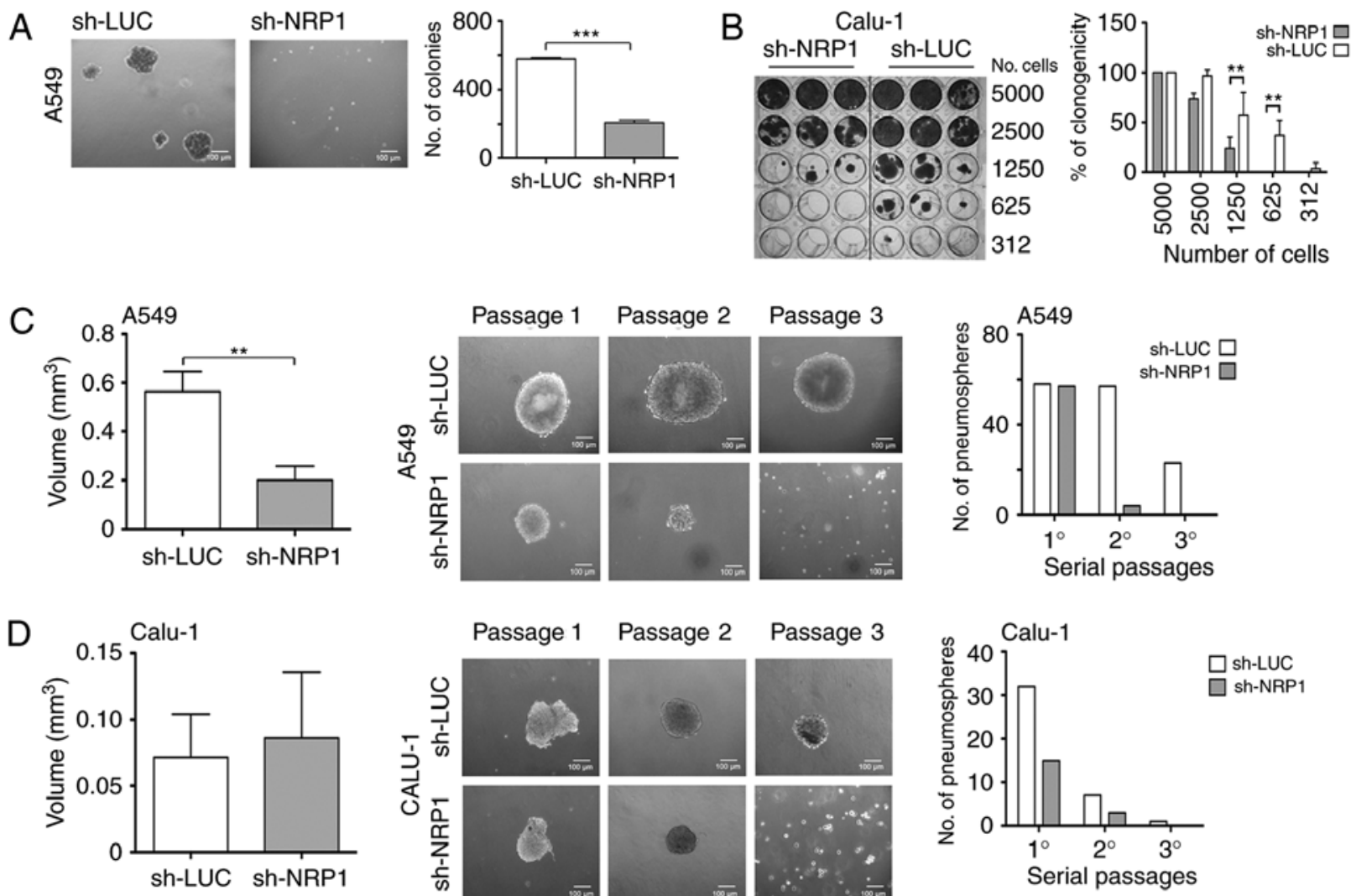
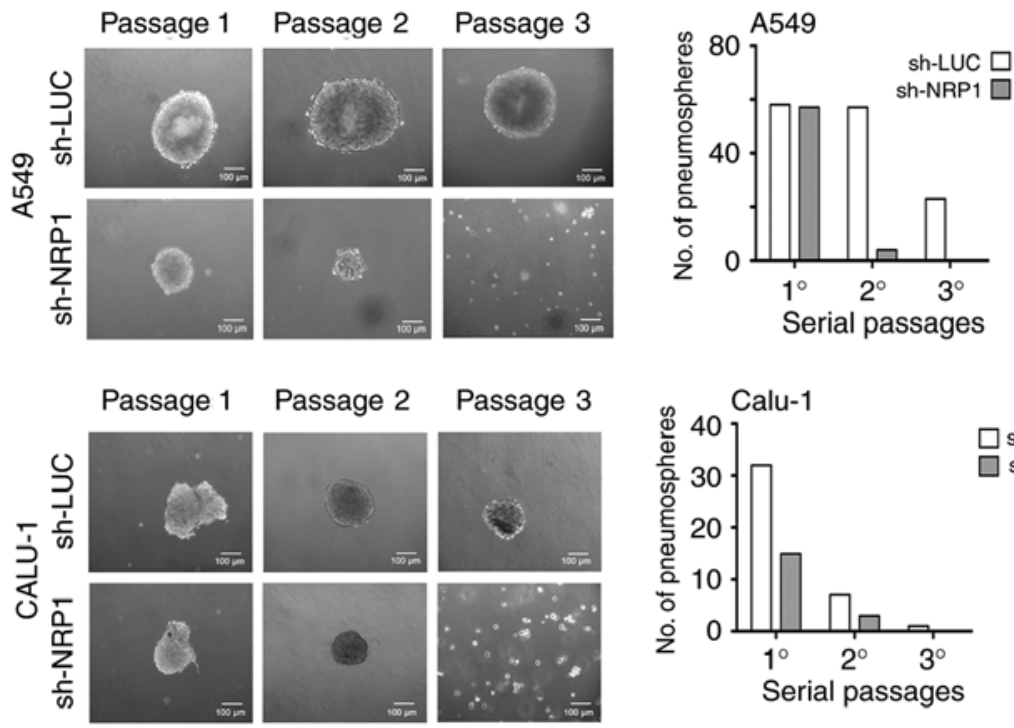

Figure 4. NRP1 expression is associated with a TIC phenotype in lung cancer cells. (A) Qualitative and quantitative representation of the number of colonies formed by sh-NRP1 A549 cells compared to control cells in soft agar $\left.{ }^{(* * * *} \mathrm{P}<0.0001\right)$. (B) Clonogenicity of Calu-1 sh-NRP1 cells compared with sh-LUC cells using a limiting dilution assay. Significant differences found in dilution 1:1250 $\left.{ }^{* *} \mathrm{P}<0.01\right)$ and 1:625 $\left({ }^{* *} \mathrm{P}<0.01\right)$. (C) Reduced pneumosphere formation in sh-NRP1 A549 cells (left panel, ${ }^{* *} \mathrm{P}<0.05$ ) and autorenewal ability in serial passages (middle and right panel). (D) Reduced pneumospheres formation in sh-NRP1 Calu-1 cells and autorenewal ability in serial passages (middle and right panel). 

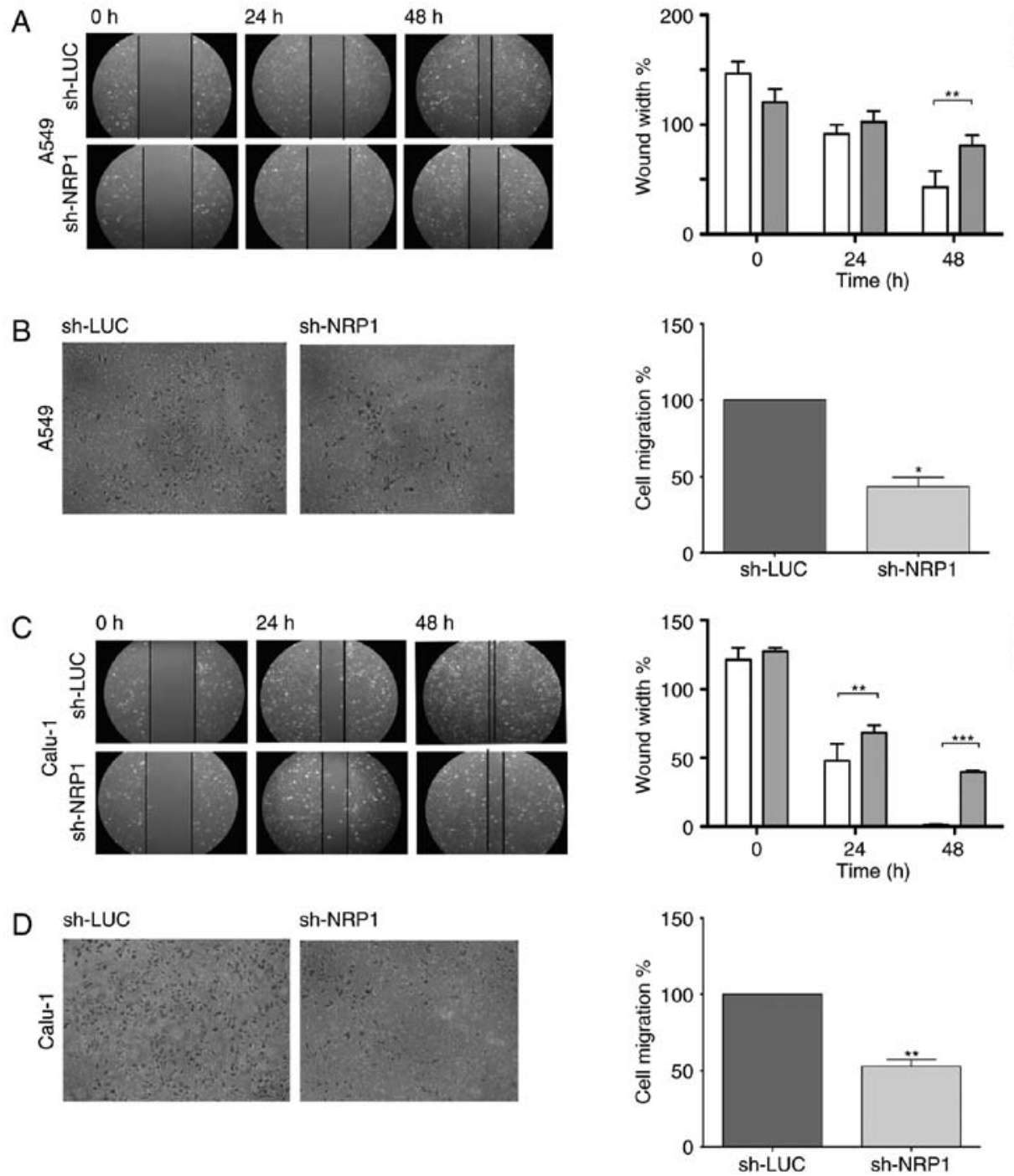

Figure 5. Inhibition of NRP1 affects cell migration. (A) Wound healing cell migration assay comparing shNRP1 and shLuc-A549 cells. (B) Effect of NRP1 knockdown on cell migration capacity evaluated in A549 cells. (C) Wound healing cell migration assay comparing shNRP1 and shLuc-Calu-1 cells. (D) Effect of NRP1 knockdown on cell migration capacity using Calu-1 cells. The graphs show 3 independent experiments (ANOVA) $\left({ }^{*} \mathrm{P}<0.01\right.$ and $\left.{ }^{* *} \mathrm{P}<0.001\right)$.

Next, we tested the effect of NRP1 knockdown on the clonogenic ability of lung cancer cells. Fig. 4A and B shows that the inhibition of NRP1 resulted in a significantly reduced number of colonies for both the A549 and Calu-1 cell lines, as measured by soft agar colony formation assays and limiting dilution clonogenic analyses.

We then analyzed whether lung cancer cells with low NRP-1 expression exhibited a compromised self-renewal ability. As shown Fig. 4C and D, middle and right panel, a lower pneumosphere forming efficiency (PFE), calculated by counting the number of spheres by serial passages, was observed in both cell lines. In A549 cells, but not in Calu-1 cells, we were able to find smaller pneumospheres after NRP1 depletion (Fig. 4C and D).

Since TICs are known to possess higher cell migration capability (27), we also analyzed whether NRP1 knockdown had an impact on cell migration. Using wound healing and Transwell cell migration assays, we detected a reduced cell migration capacity of sh-NRP1 A549 (Fig. 5A and B) and shNRP1 Calu-1 cells (Fig. 5C and D), when compared with shLuc control cells. No effect was observed for cell proliferation for any of the NRP1-knockdown cell lines (data not shown).
NRPI downregulation modifies different transcriptional networks. To identify the potential signaling pathways modulated by NRP1, we performed a genome-wide analysis in NRP1-depleted cells (using an Affymetrix microarray platform). Our analysis showed 212 deregulated transcripts (142 upregulated and 70 downregulated) (Fig. 6A), including 17 deregulated miRNAs (Fig. 6D). A network analysis using the Ingenuity Pathway Analysis (IPA) platform showed that NRP1 modulates several molecular and cellular functions, such as cell migration, cell-to-cell signaling, and drug metabolism. Fig. 6B shows the top upregulated/downregulated genes observed in these analyses, 13 of which are associated with lung cancer. Notably, CDH19 and TACC1 have been associated with a TIC phenotype. Since it has been reported that OLFML3 regulates angiogenesis via BMP4 and SMADs (28) we analyzed the expression level of these genes in the NRP1-depleted cells. Fig. 6C shows that NRP1 inhibition is clearly associated with OLMF3 downregulation. Unexpectedly, this was also accompanied by an increase in SMAD and BMP4 expression, suggesting that the OLMF3/BMP4/SMAD signaling pathway is deregulated in lung cancer cells (Fig. 6C). It has been reported 

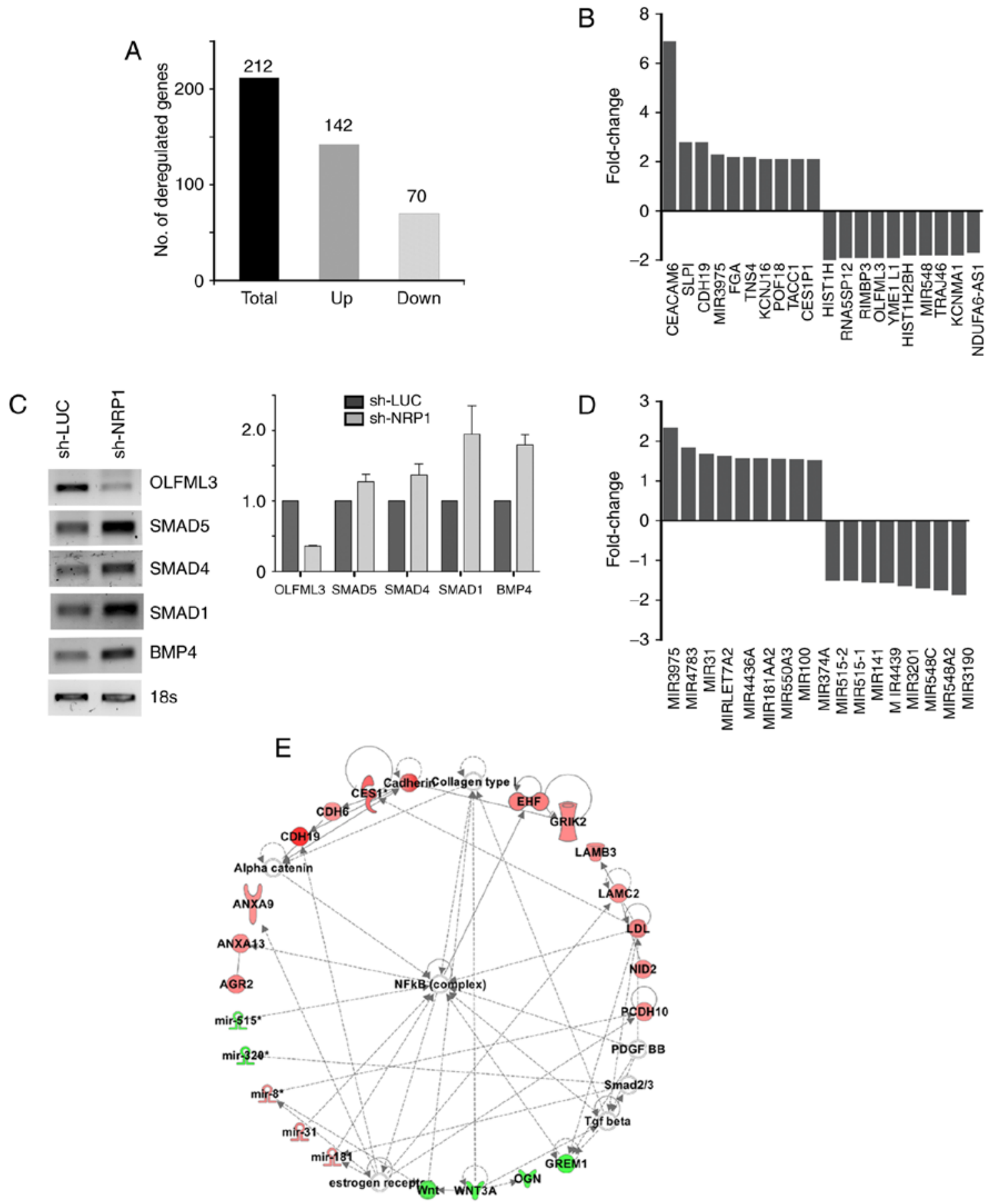

Figure 6. Inhibition of NRP1 modifies the expression of various gene networks in A549 cells. (A) Microarray analysis of sh-NRP1 A549 cells. (B) Top 10 RNAs dysregulated in sh-NRP1 cells. (C) Semi-quantitative RT-PCR of OLFML3, BMP4 and SMADs. 18s was used as a control for normalization. (D) Deregulated miRNAs in sh-NRP1 cells. (E) Dysregulated NF-KB pathway in A549 cells expressing sh-NRP1. Red colored symbols in the pathway indicate upregulation and green color denotes downregulation. Direct (solid lines) or indirect interactions (dashed lines) are shown.

that miR-31, miR-181 and miR-515 expression (which we found deregulated) is controlled by the NF- $\kappa \mathrm{B}$ signaling pathway, a master controller of the TIC phenotype (29). Supporting this, the IPA algorithm showed that the NF- $\kappa \mathrm{B}$ pathway is probably deregulated in A549-shNRP1 cells (Fig. 6E).

\section{Discussion}

NRP1 is a membrane protein expressed in embryonic $(15,30)$ and mesenchymal stem cells that regulates cell migration and proliferation (16). It has been recently demonstrated that NRP1 knockdown is sufficient to abrogate the tumor formation potential of human fibrosarcoma HT1080 cells (31). These and other reports strongly suggest that NRP-1 has a role in the regulation of a TIC phenotype $(15,16,20)$.

In the present study, we showed that $\mathrm{NRP}^{+}$lung cancer cells have a TIC phenotype. This conclusion is supported by the higher expression level of TIC markers such as OCT-4, ALDH1, p63, NANOG and EMT-associated genes (e.g. Snail). In accordance, the A549 and Calu-1 NRP1 ${ }^{+}$cell subpopulations presented 
higher clonogenic, self-renewal and cell migration abilities when compared with the NRP1 ${ }^{-}$cell subpopulations. More important, we found that NRP1 is not only a marker indicating TIC properties, but is also a key regulator of this phenotype, since RNAi loss-of-function analyses resulted in the reduced expression of molecular and phenotypic stemness markers.

Another striking result of the present study is that the p53 family member, p63 and its $\Delta \mathrm{Np} 63$ isoform, were dysregulated in NRP1 ${ }^{+}$A549 cells. These proteins are known key regulators of the TIC phenotype and EMT processes in epithelial tissue (32). Our results are further supported by Rock et al, who identified a stem cell population from human lung airway characterized by the expression of p63 (33). Furthermore, it has been reported that $\Delta \mathrm{Np} 63$ is involved in the regulation of CD44 expression, a known cancer stem cell surface marker (34-36).

The microarray analyses performed on the shNRP1-A459 cells revealed significant changes in the transcriptome profile of these cells, and among them, clear OLFML3 downregulation. Notably, we were not able to find an association of OLFML3 with BMP4 and SMAD expression in lung cancer cells, as is the case for other cell types and stimuli (28). Therefore, an alternative OLFML3 signaling pathway induced by NRP1 could be acting in these cells. Finally, it is important to note that three of the observed downregulated genes (YME1L1, KCNMA1 and $\Delta$ Np63) (37-39) are associated with mitochondrial function. Future research aimed at the association between NRP1 and mitochondrial function in tumor cells is warranted. It would be of great interest to conduct a pilot study to determine whether NRP-1/OLFML3 levels determined by qRT-PCR or immunodetection in tumor tissue obtained from biopsies, broncoalveolar fluid or serum or alternatively, miRNAs regulated by NRP-1 (miR548, miR3201 and miR3190) serve as stem cell biomarkers in lung cancer.

In conclusion, the present study demonstrated that $\mathrm{NRP} 1^{+}$ cells possess TIC properties, including the preferential expression of stemness markers, higher clonogenic and self-renewal potential, and increased cell migration capacity. These findings support an important role for NRP1 in the biology of lung cancer TICs.

\section{Acknowledgements}

We thank Raul Mojica and his staff for the microarray processing and Nelly Patiño for the flow cytometric assays (High Technology Units, National Institute of Genomic Medicine, Mexico). Luis Enrique Jiménez Hernández is a doctoral student from Programa de Doctorado en Ciencias Biomédicas, Universidad Nacional Autónoma de México (UNAM) and received fellowship 336320 from CONACyT.

\section{References}

1. Jemal A, Bray F, Center MM, Ferlay J, Ward E and Forman D: Global cancer statistics. CA Cancer J Clin 61: 69-90, 2011.

2. Sun JH, Luo Q, Liu LL and Song GB: Liver cancer stem cell markers: Progression and therapeutic implications. World J Gastroenterol 22: 3547-3557, 2016

3. Shao J, Fan W, Ma B and Wu Y: Breast cancer stem cells expressing different stem cell markers exhibit distinct biological characteristics. Mol Med Rep 14: 4991-4998, 2016.

4. Eramo A, Lotti F, Sette G, Pilozzi E, Biffoni M, Di Virgilio A, Conticello C, Ruco L, Peschle C and De Maria R: Identification and expansion of the tumorigenic lung cancer stem cell population. Cell Death Differ 15: 504-514, 2008.
5. Chen YC, Hsu HS, Chen YW, Tsai TH, How CK, Wang CY, Hung SC, Chang YL, Tsai ML, Lee YY, et al: Oct-4 expression maintained cancer stem-like properties in lung cancer-derived CD133-positive cells. PLoS One 3: e2637, 2008.

6. Tirino V, Camerlingo R, Franco R, Malanga D, La Rocca A, Viglietto G, Rocco G and Pirozzi G: The role of CD133 in the identification and characterisation of tumour-initiating cells in nonsmall-cell lung cancer. Eur J Cardiothorac Surg 36: 446-453, 2009.

7. Meng X, Li M, Wang X, Wang Y and Ma D: Both CD133' and CD133- subpopulations of A549 and H446 cells contain cancerinitiating cells. Cancer Sci 100: 1040-1046, 2009.

8. Qiu X, Wang Z,Li Y, Miao Y, Ren Y and Luan Y: Characterization of sphere-forming cells with stem-like properties from the small cell lung cancer cell line H446. Cancer Lett 323: 161-170, 2012.

9. Hongo K, Tanaka J, Tsuno NH, Kawai K, Nishikawa T, Shuno Y, Sasaki K, Kaneko M, Hiyoshi M, Sunami E, et al: CD133(-) cells, derived from a single human colon cancer cell line, are more resistant to 5-fluorouracil (FU) than CD133(+) cells, dependent on the $\beta 1$-integrin signaling. J Surg Res 175: 278-288, 2012.

10. Roudi R, Madjd Z, Ebrahimi M, Samani FS and Samadikuchaksaraei A: CD44 and CD24 cannot act as cancer stem cell markers in human lung adenocarcinoma cell line A549. Cell Mol Biol Lett 19: 23-36, 2014.

11. Xu H, Mu J, Xiao J, Wu X, Li M, Liu T and Liu X: CD24 negative lung cancer cells, possessing partial cancer stem cell properties, cannot be considered as cancer stem cells. Am J Cancer Res 6: 51-60, 2015.

12. Huo W, Du M, Pan X, Zhu X and Li Z: Prognostic value of ALDH1 expression in lung cancer: A meta-analysis. Int J Clin Exp Med 8: 2045-2051, 2015.

13. Sulpice E, Plouët J, Bergé M, Allanic D, Tobelem G and Merkulova-Rainon T: Neuropilin-1 and neuropilin-2 act as coreceptors, potentiating proangiogenic activity. Blood 111: 2036-2045, 2008.

14. Ango F: NRP1 and synapse formation. Oncotarget 7: 81975-81976, 2016.

15. Cimato T, Beers J, Ding S, Ma M, McCoy JP, Boehm M and Nabel EG: Neuropilin-1 identifies endothelial precursors in human and murine embryonic stem cells before CD34 expression. Circulation 119: 2170-2178, 2009.

16. Ball SG, Bayley C, Shuttleworth CA and Kielty CM: Neuropilin-1 regulates platelet-derived growth factor receptor signalling in mesenchymal stem cells. Biochem J 427: 29-40, 2010.

17. Hong TM, Chen YL, Wu YY, Yuan A, Chao YC, Chung YC, Wu MH, Yang SC, Pan SH, Shih JY, et al: Targeting neuropilin 1 as an antitumor strategy in lung cancer. Clin Cancer Res 13: 4759-4768, 2007.

18. Peng Y, Liu YM, Li LC, Wang LL and Wu XL: MicroRNA-338 inhibits growth, invasion and metastasis of gastric cancer by targeting NRP1 expression. PLoS One 9: e94422, 2014.

19. Cao Y, Wang L, Nandy D, Zhang Y, Basu A, Radisky D and Mukhopadhyay D: Neuropilin-1 upholds dedifferentiation and propagation phenotypes of renal cell carcinoma cells by activating Akt and sonic hedgehog axes. Cancer Res 68: 8667-8672, 2008.

20. Hamerlik P, Lathia JD, Rasmussen R, Wu Q, Bartkova J, Lee M, Moudry P, Bartek J Jr, Fischer W, Lukas J, et al: Autocrine VEGF-VEGFR2-Neuropilin-1 signaling promotes glioma stem-like cell viability and tumor growth. J Exp Med 209: 507-520, 2012.

21. Barr MP, Gray SG, Gately K, Hams E, Fallon PG, Davies AM, Richard DJ, Pidgeon GP and O'Byrne KJ: Vascular endothelial growth factor is an autocrine growth factor, signaling through neuropilin-1 in non-small cell lung cancer. Mol Cancer 14: 45, 2015.

22. Schneider CA, Rasband WS and Eliceiri KW: NIH Image to ImageJ: 25 years of image analysis. Nat Methods 9: 671-675, 2012.

23. Irizarry RA, Bolstad BM, Collin F, Cope LM, Hobbs B and Speed TP: Summaries of Affymetrix GeneChip probe level data. Nucleic Acids Res 31: e15, 2003.

24. Skirecki T, Hoser G, Kawiak J, Dziedzic D and DomagałaKulawik J: Flow cytometric analysis of CD133- and EpCAM-positive cells in the peripheral blood of patients with lung cancer. Arch Immunol Ther Exp 62: 67-75, 2014.

25. Kajstura J, Rota M, Hall SR, Hosoda T, D'Amario D, Sanada F, Zheng H, Ogórek B, Rondon-Clavo C, Ferreira-Martins J, et al: Evidence for human lung stem cells. N Engl J Med 364: 1795-1806, 2011

26. Mani SA, Guo W, Liao MJ, Eaton EN, Ayyanan A, Zhou AY, Brooks M, Reinhard F, Zhang CC, Shipitsin M, et al: The epithelial-mesenchymal transition generates cells with properties of stem cells. Cell 133: 704-715, 2008 . 
27. Lamb R, Lisanti MP, Clarke RB and Landberg G: Co-ordination of cell cycle, migration and stem cell-like activity in breast cancer. Oncotarget 5: 7833-7842, 2014.

28. Miljkovic-Licina M, Hammel P, Garrido-Urbani S, Lee BP Meguenani M, Chaabane C, Bochaton-Piallat ML and Imhof BA: Targeting olfactomedin-like 3 inhibits tumor growth by impairing angiogenesis and pericyte coverage. Mol Cancer Ther 11: 2588-2599, 2012.

29. Kastrati I, Canestrari E and Frasor J: PHLDA1 expression is controlled by an estrogen receptor-NFKB-miR-181 regulatory loop and is essential for formation of $\mathrm{ER}^{+}$mammospheres. Oncogene 34: 2309-2316, 2015.

30. Brusselmans K, Bono F, Collen D, Herbert JM, Carmeliet P and Dewerchin M: A novel role for vascular endothelial growth factor as an autocrine survival factor for embryonic stem cells during hypoxia. J Biol Chem 280: 3493-3499, 2005.

31. Misra RM, Bajaj MS and Kale VP: Vasculogenic mimicry of HT1080 tumour cells in vivo: Critical role of HIF-1 $\alpha$-neuropilin-1 axis. PLoS One 7: e50153, 2012.

32. Oh JE, Kim RH, Shin KH, Park NH and Kang MK: DeltaNp63a protein triggers epithelial-mesenchymal transition and confers stem cell properties in normal human keratinocytes. J Biol Chem 286: 38757-38767, 2011.

33. Rock JR, Onaitis MW, Rawlins EL, Lu Y, Clark CP, Xue Y, Randell SH and Hogan BL: Basal cells as stem cells of the mouse trachea and human airway epithelium. Proc Natl Acad Sci USA 106: 12771-12775, 2009.
34. Chen Y, Zhao J, Luo Y, Wang Y, Wei N and Jiang Y: Isolation and identification of cancer stem-like cells from side population of human prostate cancer cells. J Huazhong Univ Sci Technolog Med Sci 32: 697-703, 2012.

35. Leung EL, Fiscus RR, Tung JW, Tin VP, Cheng LC, Sihoe AD, Fink LM, Ma Y and Wong MP: Non-small cell lung cancer cells expressing CD44 are enriched for stem cell-like properties. PLoS One 5: e14062,2010.

36. Boldrup L, Coates PJ, Gu X and Nylander K: DeltaNp63 isoforms regulate CD44 and keratins 4, 6, 14 and 19 in squamous cell carcinoma of head and neck. J Pathol 213: 384-391, 2007.

37. Ruan Y, Li H, Zhang K, Jian F, Tang J and Song Z: Loss of Yme1L perturbates mitochondrial dynamics. Cell Death Dis 4: e896, 2013.

38. Singh H, Lu R, Bopassa JC, Meredith AL, Stefani E and Toro L: MitoBK $_{\mathrm{Ca}}$ is encoded by the Kcnmal gene, and a splicing sequence defines its mitochondrial location. Proc Natl Acad Sci USA 110: 10836-10841, 2013.

39. Matin RN, Chikh A, Chong SL, Mesher D, Graf M, Sanza' P, Senatore V, Scatolini M, Moretti F, Leigh IM, et al: p63 is an alternative p53 repressor in melanoma that confers chemoresistance and a poor prognosis. J Exp Med 210: 581-603, 2013. 